Schweizerische Landwirtschaft und Globalisierung

\title{
4. Soziale Entwicklung
}

\section{Christoph Stamm}

\section{(2) OpenEdition}

\section{Journals}

Electronic version

URL: http://journals.openedition.org/sjep/900

ISSN: 1663-9677

Publisher

Institut de hautes études internationales et du développement

Printed version

Date of publication: 1 mai 2002

Number of pages: 193-205

ISSN: 1660-5926

Electronic reference

Christoph Stamm, "4. Soziale Entwicklung », Schweizerisches Jahrbuch für Entwicklungspolitik [Online], 21 | 2002, Online erschienen am: 11 September 2012, abgerufen am 07 September 2020. URL : http:// journals.openedition.org/sjep/900 


\section{SOZIALE ENTWICKLUNG*}

\subsection{WELTKONFERENZ GEGEN RASSISMUS IN DURBAN}

Die Vereinten Nationen und Gastgeber Südafrika organisierten vom 31. August bis zum 8. September 2001 in der südafrikanischen Stadt Durban die dritte «Weltkonferenz gegen Rassismus, Rassendiskriminierung, Fremdenfeindlichkeit und damit zusammenhängende Formen der Intoleranz». Die Austragung der Konferenz im ehemaligen Apartheitsstaat Südafrika hatte hohen Symbolwert, waren doch die beiden bisherigen Konferenzen gegen Rassismus (1978, 1983) von der Debatte über die Apartheidspolitik geprägt gewesen. An der Konferenz. nahmen 163 Staaten, 12 Staatschefs und knapp 4000 Repräsentanten von Nichtregierungsorganisationen teil.

Die UNO-Generalversammlung wollte, dass sich die Konferenz aktionsorientiert mit praktischen Massnahmen zur Bekämpfung von Rassismus beschäftigt. Die Generalsekretärin der Konferenz. Mary Robinson wünschte sich im Vorfeld, dass die Opfer, die alle dasselbe Recht auf eine Anhörung hätten, zu Wort kommen, und dass alle Länder oder Regierungen, welche diese Ungleichheiten verursachen, zur Rede gestellt werden.

Ziel der Konferenz, war es, eine Schlusserklärung und ein Aktionsprogramm zur betreffenden Problematik zu verabschieden. Bereits die Länge des Konferenztitels deutete an, dass eine breite Themenliste an zu verurteilenden und zu bekämpfenden Phänomenen diskutiert werden sollte. Nach dem Willen des vorbereitenden Komitees und einzelner Staaten sollte sich die Konferenz ausserdem mit den Themen negative Einstellung gegenüber Migranten, Islamophobie, Antiarabismus, Sklaverei, Kolonialismus und Zionismus beschäftigen.

\section{$\square$ Die Frage der Wiedergutmachung von Sklavenhandel,} Sklaverei und Kolonialismus

Diese Kontroverse um die Vergangenheit war eine der Umstrittensten der Konferenz. Es gab keineswegs zwei einheitlich sich gegenüberstehende Meinungsblöcke. Eine Mehrzahl afrikanischer Staaten forderten die Anerkennung des Sklavenhandels als Verbrechen gegen die Menschlichkeit und verlangten von den Staaten, die jahrhundertelang vom transatlantischen Slavenhandel profitiert hatten, eine Entschuldigung. Andere Staaten und afrikanische und amerikanische Schwarzen-Organisationen verlangten mehr als einen symbolischen Akt. Sie verlangten Entschädigungszahlungen an die Opfer der Sklaverei. Diese Wiedergutmachungsforderungen nahmen je nach Kläger verschiedene Formen an und gingen vom Schuldenerlass für afrikanische Staaten, über die Schaffung eines Bildungsfonds bis zu Reparationszahlungen an Individuen. Dritte forderten zudem Entschädigungen für die durch Kolonialismus verursachten Schäden und Nachteile. Die am Sklavenhandel beteiligten europäischen Staaten lehnten jede Diskussion über Entschädigungszahlungen kategorisch ab und betonten, dass die

* Von Christoph Stamm, Student in Politikwissenschaft an der Universität Genf. 
Konferenz die Bekämpfung aktueller Diskriminierung zum Ziel hätte. Die Gegenseite liess dieses Argument nicht gelten, da die negativen Konsequenzen der Sklaverei bis zum heutigen Tag nachwirken würden.

\section{$\square$ Die israelisch-palästinensische Frage und die Gleichsetzung von Zionismus mit Rassismus}

Neben der Problematik der Sklaverei war das Thema des Zionismus wohl das kontroverseste. Die arabischen und einige islamische Staaten bezeichneten Israel als Apartheitsstaat und wollten im Schlussdokument Zionismus mit Rassismus gleichgesetzt sehen. Einige Nichtregierungsorganisationen klagten Israel der ethnischen Säuberung und der Kriegsverbrechen gegen das palästinensische Volk an. Die israelische Delegation bezeichnete den israelisch-palästinensischen Konflikt als politisch und nicht ethnisch und erinnerte daran, dass die Juden während Jahrhunderten Hauptopfer des Rassismus waren. Angesichts der Aussichtslosigkeit einer Kompromisslösung verliess die US-amerikanische Delegation - sie hatte sich ganz hinter Israel gestellt - die Konferenz. Auch der israelische Aussenminister Shimon Peres rief die israelische Delegation zurück. Andere Staaten, namentlich die europäische Union und Norwegen, widersetzten sich ebenfalls der Gleichsetzung von Zionismus mit Rassismus und setzten sich weiterhin für eine Kompromisslösung ein.

\section{$\square$ Die Rolle der Nichtregierungsorganisationen}

Einigen der NRO ist es zu verdanken, dass wichtige Phänomene der Rassendiskriminierung im Lärm der Debatte um die beiden Hauptstreitpunkte nicht vollständig untergingen. So warfen indische NRO ein Licht auf die Situation der Unberührbaren (Dalit). Trotz einer Gleichstellung in der indischen Verfassung sind die 170 Millionen Dalit täglicher Diskriminierung und Gewalt ausgesetzt. Es wurde verlangt, dass die Konferenz die Opfer des Kastensystems zu Wort kommen lasse und dass die «Unberührbarkeit» als Verbrechen gegen die Menschlichkeit in die Schlussdeklaration aufgenommen werde.

NRO wiesen unter anderem auf die unsägliche Situation der afghanischen Frauen, die Unterdrückung der chinesischen Minderheiten, die Lage der Weissen in Zimbabwe, die Sklaverei im Sudan und die schlechte Situation der indigenen Völker Amerikas hin. Es wurden auch allgemeinere Probleme wie die diskriminatorische Anwendung der Todesstrafe oder die besondere Gefährdung der Flüchtlinge und Asylsuchenden diskutiert. Die NRO forderten in ihrer Schlusserklärung die Staaten auf, endlich zu den Taten zu schreiten und der Krise der Menschenrechte entgegenzutreten.

Andererseits gab es aber auch einige NRO, die mit Voten der Intoleranz gegen einzelne Länder oder Gemeinschaften die Spannungen erhöhten und eine Kompromissuche erschwerten.

\section{$\square$ Die Haltung der Schweiz}

Die achtköpfige Schweizer Delegation wurde von Claudia Kaufmann, Generalsekretärin des Departements des Innern und Leiterin der Delegation an der Vorbereitungskonferenz, angeführt.

Die Schweiz hielt sich aus den kontroversen Hauptdebatten zurück und setzte sich mit ihrer «technischen Agenda» vor allem für den aktuellen und zukünfti- 
gen Kampf gegen Rassismus ein. So konnte die Schweizer Delegation die Idee der Bildung eines Observatoriums in die Diskussion einbringen. Diese aus unabhängigen Experten gebildete Institution soll die Umsetzung des Aktionsplans überwachen und dafür sorgen, dass das Papier nicht in Vergessenheit gerät. Die Schweiz setzte sich auch für die Förderung nationaler Institutionen zum Kampf gegen Rassismus und zur strafrechtlichen Verfolgung von Rassisten ein. Am Herzen lagen der Schweizer Delegation ausserdem die folgenden Themen: Kampf gegen Internetseiten mit rassistischen Inhalten, Kampf gegen Diskriminierung in Erziehung und Bildung, Schutz der Frauen vor doppelter Diskriminierung, Verhinderung der Gleichsetzung von Zionismus mit Rassismus, Gleichsetzung von Antisemitismus mit Rassimsus, Verwendung des Ausdrucks «Holocaust» ausschliesslich für die Eliminierung der Juden während der Nazidikatur, die Vertretung der Interessen der Roma, Jenischen und Sinti, das Bedauern über Sklavenhandel und Sklaverei und die Bildung eines Forums für die Diskussion über Formen der Wiedergutmachung.

\section{$\square$ Schweiz-interne Auswirkungen der Konferenz}

Die Vorbereitungen für die Weltkonferenz gegen Rassismus boten Gelegenheit, die Debatte über die Bekämpfung des Rassismus in der Schweiz wieder aufzunehmen. Sie gaben Anlass, Bilanz zu ziehen über die seit sieben Jahre bestehende Gesetzgebung gegen Diskriminierung, seit der Einführung von Artikel 261 des Strafgesetzbuches im Jahr 1994, welcher die öffentliche Anstachelung zu Rassenhass und -diskriminierung bestraft und auch heute noch das einzige Instrument zur Rassismusbekämpfung in der Schweiz bildet.

Der Sekretär des Westschweizer Verbandes Association romande contre le racisme (ACOR) stellt fest, dass dieses Instrument es nicht gestatte, Rassismuserscheinungen im Alltag, wie auch sich aus Massnahmen des Staates ergebende Formen institutionellen Rassismus wirksam zu bekämpfen. Er ruft daher zur Annahme eines allgemeinen Gesetzes gegen jegliche Form von Diskriminierung auf.

ACOR hat seinerseits eine Petition lanciert, welche die Eidgenössischen Räte ersucht, jedes Eintreten auf einen rassistischen und diskriminierenden Gesetzgebungsentwurf abzulehnen, der der internationalen UN-Konvention zur Beseitigung der Rassendiskriminierung zuwiderläuft. Die Petition fordert ferner den Bundesrat auf, den gegen die Anwendung dieser Konvention eingebrachten Vorbehalt der Schweiz zurückzuziehen. Es handelt sich hierbei um die wichtigste Kritik gegen den Entwurf eines neuen Ausländergesetzes (siehe Anfang Punkt 5.4.2). Mit dieser Petition greift ACOR den Vorschlag auf, der vom Europarat anlässlich der europäischen Konferenz in Strassburg zur Vorbereitung der Weltkonferenz in Durban eingebracht wurde und der die Mitgliedsstaaten auffordert, jeden Vorbehalt gegenüber der Anwendung der UN-Konvention aufzugeben.

In diesem Kontext hat das Eidgenössische Departement des Innern eine Fachstelle für Rassismusbekämpfung geschaffen und stellt einen Kredit von 15 Millionen Franken für Projekte zugunsten der Menschenrechte und der Prävention gegen Rassismus bereit. Hierdurch setzt das EDI die in seiner Erklärung zum Bericht der unabhängigen Expertenkommission Schweiz - Zweiter Weltkrieg angekündigten Massnahmen $\mathrm{um}^{1}$.

1. Bergier-Bericht, Die Schweiz und die Flüchtlinge zur Zeit des Nationalsozialismus; Pressemitteilungen des EDI vom Dezember 1999 und Februar 2001. 
Schliesslich ist noch zu vermerken, dass die Vorbereitung der Weltkonferenz in Durban die in der Schweiz lebenden Schwarzen veranlasst hat, sich - über ihre unterschiedliche Staatsangehörigkeit hinweg - zu einem Verband zusammenzuschliessen.

\section{$\square$ Schlusserklärung und Aktionsplan}

Nach heftigsten Debatten und einer Verlängerung der Konferenz um einen Tag einigten sich die Teilnehmer in letzter Minute doch noch auf eine Schlusserklärung und auf einen Aktionsplan. Diese beinhalten folgende Hauptaussagen und Aufforderungen:

Sklavenhandel und Sklaverei sind ein Verbrechen gegen die Menschlichkeit. Sie gehörten, zusammen mit dem Kolonialismus, zur Hauptquelle und zum Hauptausdruck von Rassismus, Rassendiskriminierung, Fremdenfeindlichkeit und damit zusammenhängenden Formen der Intoleranz. Schwarze, Asiaten und indigene Völker waren die Hauptopfer dieser Praktiken. Die Konferenz bedauert diese historischen Ungerechtigkeiten mit den bis heute wirkenden negativen Konsequenzen. Die reichen Länder, die UNO und die internationalen Finanzorganisationen sind aufgerufen, innovative Anstrengungen zum Abbau von Armut, Unterentwicklung und Marginalisierung (z.B. die «Neue Afrikanische Initiative», siehe Rahmentext am Ende des Kapitels) mit beträchtlichen zusätzlichen Finanzmitteln zu unterstützen.

Die Teilnehmer lehnen jede Rassentheorie ab und verurteilen heutige Formen der Sklaverei und des Menschenhandels. Sie fordern die Staaten auf, Massnahmen gegen diese Praktiken, die einen schlimmen Verstoss gegen die Menschenrechte darstellen, zu ergreifen.

Die Konferenz ist besorgt über die Lage im Nahen Osten und über das Aufkommen von Antisemitismus, Islamophobie und religiöser Intoleranz. Sie verurteilt rassistische und gewalttätige Gruppen, welche sich gegen jüdische, arabische und muslimische Gemeinschaften wenden. Das Recht des pälestinensischen Volkes auf Selbstbestimmung und auf einen eigenen Staat, und das Recht der Nahost-Staaten einschliesslich Israel auf Sicherheit wird anerkannt. Die Teilnehmer erinnern daran, dass der Holocaust niemals vergessen werden sollte.

Die Staaten werden aufgefordert, religiöser Intoleranz und Diskriminierung entgegenzutreten. Die Konfliktparteien im Nahen Osten werden zur Einstellung der Gewalt und zur Wiederaufnahme von Friedensverhandlungen aufgefordert. Konflikte sollen mit pazifistischen Mitteln und im politischen Dialog gelöst werden.

Die Weltkonferenz anerkennt die Rechte der indigenen Völker und der religiösen, ethnischen oder kulturellen Minderheiten. Gegen diese Gruppen bestehende institutionelle und konstitutionelle Diskriminierungen müssen abgebaut werden. Auch die Migrationspolitik und das Strafrecht der Länder müssen von rassistischen Gesetzen befreit werden.

Die Weltkonferenz betont schlussendlich, dass alle Völker und alle Individuen, ungeachtet der vielen Unterschiede, eine einzige Menschenfamilie bilden.

\section{$\square$ Bilanz}

Nachdem die Konferenz beinahe gescheitert wäre, kann der erzielte Kompromiss als Erfolg betrachtet werden. Afrikanische und arabische Staaten sowie Israel 
zeigten sich vom Schlussresultat befriedigt. Viele Teilnehmer bedauerten aber, dass die Nahostproblematik soviel Platz eingenommen und andere wichtige Themen verdrängt hatte. Die Anerkennung des Sklavenhandels als Verbrechen gegen die Menschlichkeit könnte die Tür für weitergehende Entschuldigungen und Formen der Wiedergutmachung öffnen. Die nächste Diskussion über dieses Thema müsste aber auch den arabischen und innerafrikanischen Menschenhandel mit einbeziehen. Umstritten bleibt auch das Ausmass der negativen Konsequenzen von Sklaverei und Kolonialismus. Die gegenwärtigen sozialen und wirtschaftlichen Probleme können nicht ausschliesslich dem Kolonialismus angelastet werden.

Das erarbeitete Aktionsprogramm enthält eine Reihe nützlicher Aufforderungen und Empfehlungen. Sofern diese von den einzelnen Staaten aufgegriffen und in der nationalen Gesetzgebung berücksichtigt werden, hat sich die Konferenz gelohnt.

\section{QUELLEN}

AGEFI, 29. August bis 10. September 2001.

Le Courrier, 24. August bis 10. September 2001.

Le Temps, Les Noirs de Suisse s'associent pour mieux se défendre, 13. August 2001.

Le Temps, 25. August bis 10. September 2001.

Neue Zürcher Zeitung, 24. August bis 10. September 2001.

«World Conference against Racism, Racial Discrimination, Xenophopia and Related Intolerance»: $<$ www.un.org/wcar/coverage.htm $>$ und <www.unhchr.ch/html/racism $>$.

ACOR: <www.AcorSosRacisme.org >

\subsection{AUSSERORDENTLICHE GENERALVERSAMMLUNG DER UNO ZUM THEMA AIDS}

Vom 25. bis zum 27. Juni 2001 versammelte sich die internationale Gemeinschaft zur Spezialsession über HIV/Aids in New York. Die erste Sondersession in der Geschichte der UNO, die einer Krankheit gewidmet war, brachte die Dringlichkeit des globalen Problems zum Ausdruck. Ziel der Versammlung war es, den internationalen Kampf gegen die Aids-Epidemie zu verstärken und die dafür benötigten Ressourcen zu mobilisieren.

\section{$\square$ Das Ausmass der Epidemie}

Seit der Identifizierung von HIV/Aids im Jahr 1981 sind 25 Millionen Menschen an der Immunschwächekrankheit gestorben. Von den 40 Millionen Menschen, die Ende 2001 mit dem Virus infiziert waren, hatten sich allein im Jahr 20015 Millionen Menschen neu angesteckt. Afrika südlich der Sahara ist die am meisten von der Krankheit betroffene Region. Die Anzahl der Infizierten wurde Ende 2001 auf über 28 Millionen geschätzt. Aber auch in Asien, Osteuropa und der Karibik breitet sich die Epidemie schnell aus. In einigen Ländern konnte die Ausbreitung der Epidemie gebremst werden, doch bis jetzt gelang es einzig Uganda, den Anteil der infizierten Personen gemessen an der Gesamtbevölkerung zu senken. Die in der Schweiz im Jahr 2001 gestiegene Zahl der Neuinfizierten zeigt, dass die Krankheit auch in den reichen Ländern noch keineswegs besiegt ist.

\section{$\square$ Die Auswirkungen von HIV/Aids auf die Entwicklung}

Die Aids-Epidemie stellt eine grosse Gefahr für die Entwicklung dar. Das am Milleniumsgipfel vereinbarte Ziel, die Armut bis zum Jahr 2015 zu halbieren, ist 
durch die rapide Ausbreitung der Infektionskrankheit gefährdet. Schätzungen gehen davon aus, dass die Krankheit das jährliche Pro-Kopf-Wachstum in den am schlimmsten betroffenen Ländern um bis zu 1,2\% bremst. Die Epidemie lässt die Zahl der Armen ansteigen und die Lebenserwartung (in einigen Ländern um bis zu 20 Jahre) sinken. Regierungen und Unternehmen verlieren ihre Angestellten, was das Kompetenzniveau der entsprechenden Verwaltungen und Firmen sinken lässt. Die durch die Epidemie verursachten Kosten für Gesundheit, soziale Sicherheit und Bildung übersteigen bei weitem die Mittel der betroffenen Länder und machen erreichte Fortschritte zunichte. Da auch der Landwirtschaft Ressourcen entzogen werden, kommt es in einigen Regionen zu einer Verringerung der Nahrungsproduktion.

Frauen und Mädchen tragen die Hauptlast der Epidemie. Die Frauen übernehmen die Pflege der kranken Verwandten; die Mädchen müssen zusätzliche Verantwortung in der Familie übernehmen, was sie vom Schulbesuch abhält. Aids beeinträchtigt das ganze soziale Gefüge und kann die soziale und politische Stabilität gefährden.

Die Erfahrungen der Länder, in denen die Epidemie relativ erfolgreich bekämpft wird, zeigen, dass die Errungenschaften der Entwicklung nur bewahrt werden können, wenn der Kampf gegen Aids in die Entwicklungsstrategien und -programme integriert wird. Eine effiziente Antwort auf die Krankheit verlangt die Einbindung aller staatlichen Institutionen und der Zivilgesellschaft in ein nationales Programm. Die in den relevanten Bereichen tätigen Personen müssen zudem eine angemessene Ausbildung erhalten.

\section{Schlussdeklaration}

Nach heftigen Debatten wurde die gemeinsame Verpflichtungserklärung «Weltweite Krise, weltweite Aktion » im Konsens verabschiedet. Es wird festgehalten, dass Armut und «Nichtwissen» die wichtigsten Faktoren für die Ausbreitung der Krankheit darstellen. Das Abstreiten oder das Verharmlosen des Aidsproblems und die Diskriminierung und Marginalisierung von Kranken laufen den Präventionsbemühungen entgegen und verschlimmern die Auswirkungen der Epidemie.

Der erste weltweite Plan zum Kampf gegen HIV/Aids stützt sich auf folgende Schwerpunkte: Prävention, verbesserter Zugang zu Therapie und Behandlung, Betreuung von Aids-Waisenkindern, Erweiterung der Partnerschaft zwischen privatem und öffentlichem Sektor, Notwendigkeit einer rascheren multisektoriellen Reaktion auf die Epidemie und eine Ressourcenmobilisierung, die dem Ausmass der Krise entspricht. Was die Ressourcen angeht, so verlangt der Plan während einer Mindestdauer von 10 Jahren jährliche Ausgaben von 7 bis 10 Milliarden US-\$ in den Ländern mit mittlerem und tiefem Einkommen (bis jetzt wurden jährlich zwischen 1,5 und 2 Milliarden US-\$ eingesetzt). Ein Teil dieser Kosten soll von den betroffenen Ländern selbst aufgebracht werden, der andere Teil soll aus anderen Quellen (reiche Länder, Privatsektor, internationale Organisationen, NRO) kommen. Stehen weniger Mittel zur Verfügung, so besteht die Gefahr, dass die Behandlung und Betreuung der Kranken zu kurz kommt. Laut Experten ist aber eine Doppelstrategie Prävention plus Behandlung unabdingbar.

Die Erwähnung besonders gefährdeter Gruppen (Prostituierte und deren Kunden, Drogenkonsumenten, Homosexuelle) wäre die Basis für den Aufbau spezifischer Schutzprogramme gewesen. Auf Druck religiöser Staaten und von Ländern, die 
durch konservativ-religiöse Gruppierungen beeinflusst wurden, musste jedoch auf die Benennung gefährdeter Gruppen in der Deklaration verzichtet werden. Dagegen wird erwähnt, dass die Einhaltung der Menschenrechte, die Gleichstellung von Mann und Frau und die Verhinderung sexueller Gewalt gegen Frauen eine wichtige Basis für eine erfolgreiche Aidsprävention darstellen.

Die Schweizer Delegation beteiligte sich unter der Leitung von Bundesrätin Ruth Dreifuss an der Konferenz. Ruth Dreifuss appellierte an die internationale Gemeinschaft, die Mittel zum Kampf gegen das Immunschwächevirus zu erhöhen. Die Schweiz wird ihren Beitrag an UNAIDS und an andere in der Aidsbekämpfung tätige internationale Organisationen zwischen 2000 und 2002 auf 8 Millionen Franken vervierfachen. Ausserdem forderte die Bundesrätin eine drastische Senkung der Preise für Aids-Medikamente.

\section{$\square$ Globaler Aids- und Gesundheitsfonds ${ }^{2}$}

Dieser mit mehreren Milliarden US-\$ zu dotierende Fonds soll der Bekämpfung von HIV/Aids, Tuberkulose und Malaria dienen. In einer Rede anlässlich der Weltgesundheitsversammlung nannte Kofi Annan die verheerenden Auswirkungen dieser Krankheiten als eines der Haupthindernisse für Entwicklung.

Der bis Ende 2001 zu bildende Fonds wird von einem unabhängigen Organ geleitet werden. Er soll weder bestehende Finanzierungsmechanismen für Programme gegen Aids, Malaria und Tuberkulose ersetzen, noch die Anstrengungen und Investitionen der Entwicklungsländer verdrängen. Der Fonds wird daher aus neuen und zusätzlichen Mitteln konstituiert werden. Staaten, Firmen und Privatpersonen werden aufgefordert, sich an der Finanzierung beteiligen. Bis Ende 2001 wurden Spenden von knapp 1,5 Milliarden US-\$ gesprochen. Im Jahr 2002 soll eine öffentliche Kampagne zur Mittelbeschaffung organisiert werden.

\section{$\square$ Die Debatte um den Zugang zu Medikamenten}

Obwohl die Preispolitik nur einen der vielen Aspekte im Kampf gegen Aids darstellt, steht die Debatte über Medikamentenpreise oft im Zentrum der Diskussio- 1 nen. Diese sind, da zwei der weltgrössten Pharmakonzerne und deren Lobby in der Schweiz beheimatet sind, für unser Land von besonderer Brisanz.

In den Industrieländern belaufen sich die jährlichen reinen Medikamentenkosten für die Behandlung einer mit dem Virus infizierten Person auf ca. 12'000 SFR. Die antiretrovirale Behandlung aller 40 Millionen Infizierten würde demnach astronomische Geldsummen verschlingen. Für die armen Länder ist es daher ausserordentlich wichtig, dass Medikamente zu einem massiv tieferen Preis erhältlich sind. ${ }^{3}$ Die Debatte spielt sich auf zwei Ebenen ab. Einerseits geht es um die Preissenkung der von den Pharmamultis angebotenen Medikamente; andererseits geht es um die Möglichkeit, billige Generika in den Ländern des Südens produzieren und in andere südliche Länder exportieren zu können. Hier handelt es sich um einen Streit um Patentrechte, die im Rahmen der WTO durch das TRIPS-Abkommen geregelt werden.

D JSDW 2002, Kapitel 2.4.

2. Nach letzten Informationen wird der offizielle Name des Fonds wie folgt lauten: «Global Fund to fight aids, tuberculosis and malaria» (GF-ATM).

3. Selbst bei Tiefstpreisen (z.B. Kosten von 1 SFR pro Person und pro Tag) würde die Gefahr, dass die Aidsbekämpfung auf Kosten anderer Aufgaben im Gesundheitsbereich geht, weiterhin bestehen. 
Die Pharmaunternehmen wehren sich mit verschiedensten Argumenten gegen Lösungen, die ihre Gewinne schmälern könnten. Ein Argument ist die Resistenzbildung der Krankheitserreger, hervorgerufen durch eine unsachgemässe Anwendung der Medikamente. Ein anderes ist dasjenige der hohen Entwicklungskosten für zukünftige Medikamente: Wird der Patentschutz zu stark abgeschwächt, so schwindet auch das Investitionsinteresse für Neuentwicklungen. Die Medikamentenfirmen stehen aber unter zunehmendem Druck und sind teilweise bereit, Kompromisse einzugehen.

QUELLEN

AGEFI, 28. Juni 2001.

Le Courrier, 22. Juni 2001.

Le Temps, 22. Juni 2001.

NGLS Roundup Nr. 76, August 2001.

«Joint United Nations Programme on HIV/AIDS $»:<w w w . u n a i d s . o r g>$.

«General Assembly Special Session on HIV/AIDS » : <www.un.org/ga/aids/coverage>.

«Global Aids and Health Fond»: <www.unfoundation.org/campaigns/aids>.

\subsection{AGENDA DER INTERNATIONALEN KONFERENZEN}

\section{Session der UNO-Menschenrechtskommission}

Die Kommission für Menschenrechte der Vereinten Nationen hielt ihre 57. Session vom 19. März bis zum 27. April 2001 in Genf ab. Im Kontext der bevorstehenden Konferenz gegen Rassismus standen Diskussionen über Toleranz und Respekt auf der Tagesordnung. Am ersten Sitzungstag erklärte die Kommissarin für Menschenrechte Mary Robinson, dass sie für ein zweites Mandat nicht zur Verfügung stehe. Für den Rücktritt seien vor allem der mangelnde Rückhalt und die ungenügenden Ressourcen verantwortlich. Weniger als $2 \%$ des Budgets der UNO wird für die Förderung der Menschenrechte eingesetzt.

Dieses Jahr wurde über die Menschenrechtssituation in 22 Ländern und Regionen diskutiert. Im Vordergrund standen dabei Staaten, in denen die Menschenrechte aufgrund von Kriegssituationen besonders stark unter Druck geraten.

Bei der Verabschiedung verschiedener thematischer Resolutionen kamen die gegensätzlichen Standpunkte der Industrie- und Entwicklungsländer klar zum Vorschein. Während die westlichen Staaten sich vor allem für die Stärkung der politischen Grundrechte einsetzten, bemühten sich die Länder der Dritten Welt um die Stärkung des Rechts auf Entwicklung. Unter den verabschiedeten Resolutionen befinden sich solche in den Bereichen Menschenrechte und Globalisierung, Recht auf Entwicklung, auf Wohnraum, auf Aids-Medikamente, auf Erziehung, Rechte der Frauen und Kinder und Recht auf Nahrung.

Das Mandat des Schweizers Jean Ziegler, Sonderberichterstatter für das Recht auf Nahrung, wurde ausgedehnt, so dass es künftig auch das Recht auf Wasser umfasst.

Hervorzuheben sind zudem eine Resolution zur Ernennung eines Sonderberichterstatters für indigene Völker und eine Resolution zur Abschaffung der Todesstrafe.

Bei der Wahl neuer Kommissionsmitglieder wurde die USA nicht wiedergewählt. Dies ist das erste Mal seit der Gründung der Kommission 1947, dass das Land nicht Mitglied ist. 


\section{QUELLEN}

Le Temps, 19. April 2001, 28. April 2001.

Neue Zürcher Zeitung, 20. März 2001, 20. April 2001, 28. April 2001.

Office of the High Commissioner of Human Righst: <www.unhchr.ch>.

«57th Session of the Commission on Human Rights»: <www.unhchr.ch/htm1/menu2/2/57chr>.

\section{$\square$ Weltgesundheitsversammlung der WHO}

Die 54. Weltgesundheitsversammlung fand vom 14. bis zum 22. Mai 2001 in Genf statt. Im Zentrum der Versammlung standen die Themen Bekämpfung von HIV/Aids, Säuglingsernährung, Eindämmung des Tabakkonsums sowie die Sensibilisierung der Staaten für eine aktive Gesundheitsförderung. Die Generaldirektorin der Weltgesundheitsorganisation Gro Harlem Brundtland rief zur Überwindung der durch Armut verursachten und begünstigten Krankheiten auf.

Eine Resolution beschäftigte sich mit dem Zugang zu Medikamenten. Es wurde hervorgehoben, dass ein Drittel der Weltbevölkerung keinen Zugang zu den notwendigsten Medikamenten hat. Dieser Mangel soll unter anderem mit Preiskontrollen behoben werden. Ausserdem sollen die Forscher ermutigt werden, nach besseren Medikamenten gegen Krankheiten, die vor allem die arme Bevölkerung treffen, zu forschen.

Eine umfassende Resolution über die Ernährung des Säuglings und des Kleinkindes fordert unter anderem die 191 Mitgliedsstaaten auf, das exklusive Stillen des Säuglings in den ersten sechs Monaten zu schützen und zu fördern.

Ausserdem haben die Mitgliedsländer für mehr Wachsamkeit gegenüber der Tabakindustrie aufgerufen. Diese habe jahrelang alle Bemühungen der Regierungen und der WHO im Kampf gegen den Tabakkonsum untergraben. Die bisher ungenügende Reglementation der Tabakprodukte zum Schutz der öffentlichen Gesundheit soll verbessert werden.

Da die Schweiz bis 2002 im Exekutivrat vertreten ist, konnte sie die Themen der Versammlung in der Vorbereitungskonferenz aktiv mitgestalten.

Die Schweizer Delegation unter der Leitung von Ruth Dreifuss engagierte sich insbesondere bei der Tabakkontrolle und der Reform des Exekutivrates. Da aber ein Vorschlag zum Thema Transparenz im Tabakkontrollprozess bei anderen Delegationen auf vehementen Widerstand stiess, verzichtete die Schweiz darauf, den Vorschlag formell einzureichen. Auf Schweizer Initiative wurde zudem ein Paragraph aus dem Resolutionsentwurf über den Zugang zu Medikamenten entfernt. Der Paragraph - er hätte die WHO zur Förderung der lokalen Produktion von Medikamenten aufgefordert - wäre im Widerspruch zum TRIPS-Abkommen der WTO gestanden.

QUELLE

World Health Organization: <www.who.int>.

\section{$\square$ Internationale Arbeitskonferenz}

Vom 5. bis zum 21. Juni 2001 fand die 89. Session der Internationalen Arbeitsorganisation (ILO) in Genf statt. Die Jahreskonferenz ist die internationale Tribüne für die Diskussion sozialer und arbeitsspezifischer Fragen und Probleme. Hier wird über internationale Arbeitsnormen, Leitlinien und das Budget der ILO entschieden. Laut J. J. Elmiger, Schweizer Botschafter, spielt die Internationale 
Arbeitsorganisation die Rolle der «Beschützerin der sozialen Dimension der Globalisierung ».

Jeder der 175 Mitgliedsstaaten kann vier Delegierte an die Konferenz entsenden (2 Regierungs-, 1 Arbeitgeber- und 1 Arbeitnehmervertreter). Während den drei Konferenzwochen wurden folgende Schwerpunkte diskutiert: Eliminierung aller Formen der Zwangsarbeit, Arbeitssicherheit und -gesundheit in der Landwirtschaft, Förderung der Genossenschaften und die Zukunft der sozialen Sicherheit.

Generaldirektor Juan Somavia betonte in seinem Bericht die Wichtigkeit der Weiterentwicklung der Agenda für «Angemessene Arbeit» (Decent Work). Es gelte nun von den Aspirationen und Konzepten zu den Aktionen und Realisierungen zu schreiten. Mit der Ausbreitung humaner Arbeit könne Armut bekämpft und der Weltwirtschaft eine grössere Legitimität gegeben werden. Auch die Schweiz plädiert für dieses Anliegen und für die dazu benötigten Mittel. Sie wird ihren Beitrag an die ILO für die Jahre 2002-03 um 5\% auf knapp 4 Millionen Franken erhöhen. Dies entspricht 1,3\% des ILO-Budgets.

Anlässlich der Spezialsession zum Stand der Umsetzung der Übereinkommen wurde die Lage in 24 Ländern untersucht. Neben Fortschritten in Portugal und der Türkei stellte man auch grosse Mängel fest: In den Ländern Weissrussland, Kolumbien, Venezuela und Äthiopien wird das Übereinkommen Nr. 87 über die Gewerkschaftsfreiheit missachtet. In Myanmar und im Sudan dagegen existiert Zwangsarbeit in grossem Ausmass; das Übereinkommen Nr. 29 - es verbietet jegliche Form von Zwangsarbeit - wird missachtet. Ein erhöhter Druck auf Myanmar soll das Land zum Einlenken bewegen.

Zum ersten Mal wurden internationale Normen zur Arbeitssicherheit und -gesundheit in der Landwirtschaft verabschiedet. Zusammen mit der Bergindustrie und der Bauwirtschaft gehört die Landwirtschaft $\mathrm{zu}$ den gefährlichsten Wirtschaftssektoren. Jedes Jahr sterben 1,2 Millionen Menschen infolge von Arbeitsunfällen, die Hälfte davon in der Landwirtschaft. Die meisten Unfälle geschehen im Umgang mit giftigen chemischen Stoffen und mit Maschinen.

Die wichtigsten Punkte des Übereinkommens sind die Bereitstellung geeigneter Mittel zur Risikoeinschätzung; Präventiv- und Schutzmassnahmen für die Sicherheit im Umgang mit Maschinen, Chemieprodukten und Tieren, für die Sicherheit von Lagerung und Transport und für die Sicherheit im Bau und Unterhalt von Landwirtschaftsinstallationen; ein Versicherungsschutz für Landwirtschaftsarbeiter gegen Unfall, Krankheit und Todesfall. Dieses Übereinkommen gilt nur für Agrarangestellte, nicht aber für selbstständige Bauern. Die Schweizer Delegierten stimmten diesem mit grosser Mehrheit angenommenen Übereinkommen zu.

In der Diskussion über ein internationales Übereinkommen zur Förderung von autonomen unternehmerischen Genossenschaften wurde betont, dass diese eine wichtige Rolle bei der Schaffung von Arbeitsplätzen spielen. Auch für die erleichterte Kapitalbeschaffung, die gegenseitige Hilfe und den Aufbau eines sozialen Netzes können Genossenschaften einen wichtigen Beitrag leisten.

Vielen Mitgliedsstaaten ist die staatliche Sozial- und Krankenversicherung ein wichtiges Anliegen. Die meisten waren sich einig, dass das Ziel der Sozialpolitik die Ausweitung des Schutzes auf die ganze Bevölkerung sein soll. Die staatliche Sozial- und Krankenversicherung helfe mit, die Produktivität zu erhöhen, und stelle daher einen Nutzen für die wirtschaftliche Entwicklung dar. 
Die wichtigste Sorge der Industrieländer ist in diesem Zusammenhang die Finanzierung des Rentensystems bei einem wachsenden Anteil der alten Menschen an der Bevölkerung, währenddessen bei vielen Entwicklungsländern die Probleme im Zusammenhang mit der HIV/Aids-Epidemie im Zentrum stehen.

Das ILO-Programm zur Bekämpfung der Kinderarbeit (International Programme on the Elimination of Child Labor IPEC) - die Schweiz gehört seit $1997 \mathrm{zu}$ den 25 Geberstaaten - lancierte eine neue Initiative mit dem Ziel, die schlimmsten Formen der Kinderarbeit (Abfalltrennung, Lastentragen, Arbeit in Minen, Fischerei und kommerzielle Landwirtschaft, Prostitution und Schuldknechtschaft) in den Ländern Tansania, El Salvador und Nepal bis in zehn Jahren zu eliminieren. Andere Staaten sollen sich der Initiative anschliessen.

Der Bundesrat gab bekannt, dass die Schweiz das an der letzten Konferenz verabschiedete Übereinkommen Nr. 183 über die Mutterschaft nicht ratifizieren kann, da die nationalen gesetzlichen Leistungen bei Mutterschaft den Bestimmungen des Übereinkommens nicht genügen.

【SDW 2000, S. 205.

\section{QUELLEN}

NGLS Go Between Nr. 87, 89th International Labour Conference, Genf, Juli-August 2001. International Labour Organization: <www.ilo.org $>$.

«International Labour Conference 89th Session»: <www.ilo.org/public/english/standards/relm/ilc/ilc89>.

\section{$\square$ Nachfolgekonferenz der UNO über menschliche Siedlungen (Istanbul+5)}

Vom 6. bis zum 8. Juni 2001, fünf Jahre nach der Konferenz über menschliche Siedlungen (Habitat II), versammelten sich die UNO-Mitglieder zur Spezialsession «Istanbul+5» in New York. Habitat II markierte seinerzeit einen fundamentalen Richtungswechsel in der Urbanisierungsfrage, der sich dadurch auszeichnete, dass man anstatt des unerreichbaren Ziels der Verlangsamung der Urbanisierung die bessere Planung derselben auf die Agenda setzte.

[JSDW 1997, S. 67-73.

Fünf Jahre nach der Verabschiedung der Habitat-Agenda «Für eine angemessene Unterkunft für alle» sollte Bilanz gezogen und überprüft werden, ob und wie die Anliegen der Agenda umgesetzt werden, welche Hindernisse bestehen und welche weitergehenden Aktionen allenfalls nötig sind. Um die Fortschritte zu evaluieren, mussten die Regierungen Berichte über das in den letzten fünf Jahren Geleistete vorlegen.

Istanbul +5 konnte keine spektakulären Fortschritte erzielen. Die Bestätigung der Verpflichtungen von Habitat II, die Debatten über die wichtigsten Probleme der Urbanisierung und die Verabschiedung der «Deklaration über die Städte und andere menschliche Siedlungen im neuen Jahrtausend » bilden die Hauptresultate der Konferenz.

Seit 1996 hat sich die Urbanisierung noch einmal beschleunigt. Ungleichheiten zwischen und innerhalb von Städten verstärkten sich; die Lebensqualität in den Städten hat sich tendenziell verschlechtert. Als wichtigste Dimension der Deklaration wird die sich ausbreitende städtische Armut angesehen. Vor allem in den Entwicklungsländern sind zusätzliche Massnahmen und Mittel erforderlich, um dieses Problem zu entschärfen. 
Ein Schwerpunkt der Debatte war die Gemeindeautonomie. Dezentrale Strukuren wurden als entwicklungsfördernd anerkannt. Ein Projekt für eine internationale Charta der lokalen Autonomie wurde aber, da von einer Mehrheit als zu grosse Einmischung in nationale Angelegenheiten empfunden, abgelehnt.

Von der Schweizer Delegation wurde bemängelt, dass das in Istanbul als innovativ bezeichnete Konzept der «Partnerschaft» (die Einbindung privater Akteure und der Zivilgesellschaft in den politischen Entscheidungsfindungsprozess) von den Vereinten Nationen nur schlecht akzeptiert wird. Eine solche Partnerschaft lässt sich oft auf lokaler Ebene beobachten, Vorschläge und Entscheidungen auf höherer Ebene werden aber immer noch vorwiegend vom «Klub der Nationen» unter Ausschluss der Zivilgesellschaft gefasst. Die an dieser Konferenz zu beobachtenden Widerstände gegen eine Einbindung der Zivilgesellschaft stellen somit einen Widerspruch und einen Rückschritt dar. Die eigentliche Herausforderung in den nächsten Jahren sei daher, so die Schweizer Delegation, die Diskrepanz zwischen den internationalen Entscheidungen und den konkreten lokalen Aktionen zu verringern, um eine grössere Kohärenz und Effizienz in der Städteentwicklung zu erreichen.

Die Nichtregierungsorganisationen waren enttäuscht, dass in der Deklaration ein «Recht auf eine angemessene Unterkunft» keine Erwähnung fand. Dies stelle einen Rückschritt gegenüber der Habitat II-Konferenz dar.

\section{QUELLEN}

NGLS Roundup Nr. 79, Istanbul+5: UN General Assembly Review of the Habitat Agenda, Genf, August 2001.

United Nations Center for Human Settlements (Habitat) : <www.unchs.org $>$.

«United Nations General Assembly Special Session Istanbul +5»: <www.un.org/ga/habitat $>$.

\section{$\square$ Jahresversammlung des UNO-Wirtschafts- und Sozialrates}

In Genf traf sich vom 2. bis zum 27. Juli 2001 der ECOSOC zur Jahresversammlung. Der Wirtschafts- und Sozialrat - ihm obliegt die Koordination aller sozialen und wirtschaftlichen Aktivitäten der UNO - ist eines der Hauptorgane des UNO-Systems und besteht aus 54 Mitgliedern, die für drei Jahre gewählt sind.

Hauptthema war die internationale Hilfe für Afrika. Die UNO konstatierte, dass in den 90er Jahren Kriege, die Aids-Epidemie und die Last der Auslandschulden die in den 80er Jahren erreichten Fortschritte in Afrika zunichte gemacht haben. Auch die internationale Hilfe war in den 90er Jahren stark rückläufig.

Um das am letztjährigen Milleniumsgipfel vereinbarte Ziel der Halbierung der Armut bis $2015 \mathrm{zu}$ erreichen, bräuchte es in Afrika ein jährliches Wirtschaftswachstum von 7 bis $8 \%$. Ohne spektakuläre Verbesserung der Rahmenbedingungen könne dieses Ziel nicht erreicht werden. UNO-Generalsekretär Kofi Annan forderte deshalb eine neue, auf die Bedürfnisse der ärmsten Länder ausgerichtete Welthandelsrunde und betonte die Wichtigkeit offener Märkte des Nordens für afrikanische Produkte. Die verabschiedete Deklaration rief die Vereinten Nationen und die internationale Gemeinschaft auf, die «Neue afrikanische Initiative» (siehe Rahmentext) zu unterstützen. 


\section{QUELLEN}

Le Courrier, 3. Juli 2001.

NGLS Roundup Nr. 77, August 2001.

Economic and Social Council: <www.un.org/documents/ecosoc.htm und www.un.org/esa/coordination/ ecosoc>.

\section{Neue afrikanische Initiative}

Im Oktober 2001 lancierten mehrere afrikanische Staats- und Regierungschefs in der nigerianischen Hauptstadt Abuja die "Neue Partnerschaft für afrikanische Entwicklung (New Partnership for African Developement, Nepad). Dieser unter dem Namen "Neue afrikanische Initiative" bekannt gewordene ambitiöse Plan war von den Präsidenten Nigerias, Südafrikas, Senegals und Algeriens ausgearbeitet und am Gipfel der Organisation für Afrikanische Einheit in Lusaka und am G-8-Gipfel in Genua vorgestellt worden. Der Plan soll die Armut besiegen, indem Afrika auf einen Kurs beschleunigten Wachstums und nachhaltiger Entwicklung gebracht wird. Dabei sieht die Nepad grosse Investitionen in den Bereichen Erziehung, Infrastruktur und Computertechnologie sowie verstärkte gemeinsame Anstrengungen für Frieden und Demokratie vor. Neu an diesem Plan ist vor allem der Wille afrikanischer Führer, selber die Initiative zu ergreifen und den Schwerpunkt auf Partnerschaft statt auf Hilfe zu legen. Als nächster konkreter Schritt ist die Einrichtung eines Nepad-Sekretariats in Pretoria und eines Umsetzungsausschusses geplant.

\section{QUELLEN}

Neue Zürcher Zeitung, Zürich, 25. Oktober 2001

"The new Partnership for African Developement (Nepad) incorporating the OMEGA Plan» : 〈www.mapstrategy.com>.

\section{$\square$ Internationale Erziehungskonferenz}

Vom 5. bis zum 8. September 2001 fand in Genf die vom Internationalen Bildungsbüro (IBE) der UNESCO organisierte, internationale Erziehungskonferenz statt. Die Konferenz stand unter dem Motto «Das Zusammenleben lernen» und beschäftigte sich mit Lerninhalten und -strategien, Problemen und Lösungen. Nachdem auf der quantitativen Ebene (Einschulung möglichst aller Kinder) Fortschritte erzielt worden seien, gehe es nun darum, die Qualität der Bildung zu erhöhen.

Um das Zusammenleben zu lernen, soll in den Schulen ein Klima der Toleranz und des gegenseitigen Respektes gefördert werden. Der nach lebhaften Diskussionen in thematischen Workshops und im Plenum verabschiedete Schlussbericht betonte, dass die Akteure im Bildungswesen durch die wissenschaftlichen und technischen Fortschritte, das Zusammenleben der Kulturen und die Globalisierung vor grosse Herausforderungen gestellt sind. Die durchzuführenden Reformen sollen als Prozesse und nicht als Resultate interpretiert werden.

Den Delegierten war bewusst, dass die Schule die gesellschaftlichen Probleme nicht alleine lösen kann. Sie riefen darum zum Dialog und zur Zusammenarbeit mit allen gesellschaftlichen Akteuren auf.

\section{QUELLEN}

International Bureau of Education: <www.ibe.unesco.org>.

«46th Session of the International Conference on Education»: www.ibe.unesco.org/international/ice/ 46english/46menue.htm>. 